\title{
An analytic self-gravitating disk model: inferences and logical structure
}

\author{
R.E. Wilson \\ Astronomy Department, University of Florida, Gainesville, FL 32611, USA
}

Received: October 21, 2019; Accepted: January 10, 2020

\begin{abstract}
The foundations of an analytic Accretion-Decretion (A-D) disk model, based on equipotential theory, for mass-transfer binaries are examined. Gravitation of stars 1 and 2 and the disk, as well as disk and star rotation, are included and relevant morphology is explored. Expected applications are to disks with morphologically significant mass and substantial optical thickness. Anticipated targets include classical novae, nova-like variables, and W Serpentis binaries, with the concept invoking knowledge about Be stars and the classically strange binary $\beta$ Lyrae. The model's ideas and resulting character differ from those usually applied to optically thick disks - for example there is no need to truncate the model arbitrarily at an outer or inner limit, because it closes naturally at both places. The disk is a volume emitter with attenuation of internally generated light. Computations intrinsically produce phenomena that are characteristic of circumstellar disks in binaries - in particular tidal and rotational gravity brightening and an outer effective gravity null point that do not occur in the common axisymmetric disk model. Impersonal analysis in terms of the model (Least Squares criterion) is applied to light curves of recurrent nova CI Aquilae.
\end{abstract}

Key words: binaries: eclipsing, binaries: spectroscopic, stars: $\beta$ Lyr, CI Aql

\section{Introduction}

Prior to a half-century ago, a school of thinking disbelieved in overcontact (OC) binaries (W UMas and more massive OCs), apparently because computation of their light curves was impractical with existing computers. The likely thinking was, "If we can't do the computations, an OC model is beyond consideration." This mental barrier delayed meaningful progress on W UMas and other OCs because the adopted morphology (detached or at most point-contact) did not match the reality of overcontact (see Sec. 4.3 of Wilson, 1994, for discussion). But what do OC binaries have to do with disks? The connection is the analogous developmental situation for significantly massive disks in tight binaries disbelief in self-gravitating (i.e. significantly massive, SG) disks for lack of a way to do the proper computations (light curves, radial velocities, ...). However the point at issue is not that most disks in binaries are significantly massive - just that some very interesting ones may be, and that possibility needs investigation. 


\section{Binary morphology - role of limiting lobes}

Early thoughts on semi-detached and detached morphology concerned the strange and still not thoroughly understood object $\beta$ Lyrae (Kuiper, 1941), with circumstances of those two conditions later quantified by Kopal (Kopal, 1955, 1959). Overcontact morphology was treated by Lucy (1968a,b) and soon after by several others such as Mochnacki \& Doughty (1972a), Mochnacki \& Doughty (1972b), Mauder (1972), Lucy (1973), Wilson \& Devinney (1973), Rucinski (1973) and Rucinski (1974). The fourth morphological type, which logically involves super-synchronous rotation for at least one of the component stars, has both stars accurately filling their lobes and was defined by Wilson (1979). Examples of double contact systems are not easy to authenticate, but claimed identifications continue to appear, as by Wilson et al. (1985), Terrell (2005), Linnell et al. (2006), Terrell \& Nelson (2014), Çakirli et al. (2015) and Palma et al. (2016). See Wilson (2001) for a brief history of the four categories.

Quantitative modeling for analysis of globally self-gravitating disks in binaries begins with consideration of the relevant morphology. Figuratively one can say, "...In the garden of binaries, conceptual morphology is the gateway and analytic morphology is the pathway - so enter the gateway and follow the pathway." In other words, morphology is fundamental to understanding and to computation. So is there a preferred morphology for production of massive disks? What about double contact, which can be suspected because mass transfer creates the essential conditions as natural consequences of large scale or long-continued mass transfer. That is, transfer spins up an accreting star so as to inhibit easy net flow from disk to star. "Inhibit" does not mean "stop" and modest slowing of accretion may suffice to cause back-up of disk matter.

\section{An alternative to essentially massless disk models}

Many examples of the truncated disks currently applied are illustrated in the literature, for example Fig. 2 of Linnell and Hubeny (1996) and Fig. 1 of Hachisu and Kato (1999). Although at least several computer programs are represented by such published illustrations, their essential disk models are nearly the same, with the main differences being in parameter values, presence or absence of a splash from the impacting stream, and ways of tiling the disk surface. Commonalities in these contributions are assumptions that disk masses are small enough not to affect disk figures, and abrupt truncation on the outside so as not to come unduly close to the companion. Another is that all observable disk light comes from its surface, while almost all companion star light that encounters the disk is completely blocked (not just attenuated). The sharply defined edges of such optically thick disks may be a good match to reality in some or even most of the chosen objects. However allowance for possible semi-transparent edges would seem a prudent strategy as we move forward, because many disks are known to be optically thin and intermediate examples can be expected. Truncated 

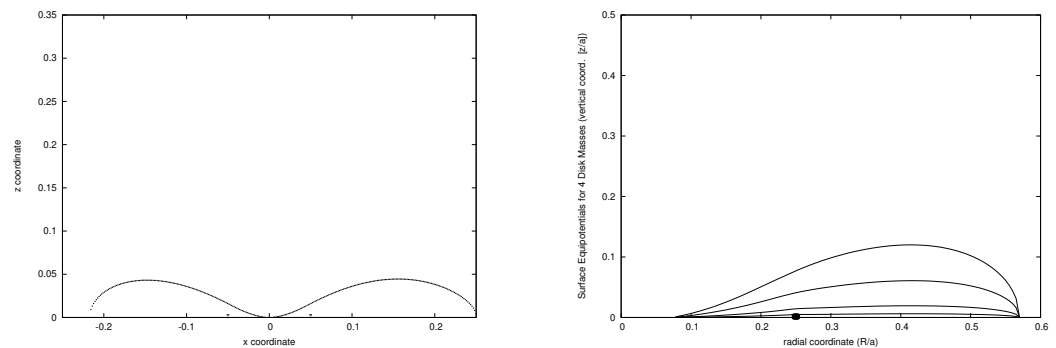

Figure 1. Left panel: a cut through CI Aql's disk surface equipotential in the $x, z$ plane at $y=0$, showing the tidal stretching that leads to several observable effects. Right panel: the $\beta$ Lyr disk surface equipotentials at positive $x$ for (disk mass)/(star mass) of 0.0400, 0.0100, 0.0010, and 0.0001. More massive $\longrightarrow$ larger $z$ height.

massless disk models have been useful to demonstrate essential optical and geometrical thickness of selected CV disks, while incorporation of self-gravity, disk tides, disk gravity brightening, surface semi-transparency, and double contact morphology can now explore previously neglected features. The disk model applied here (Wilson, 2018) is a volume (not surface) emitter whose figure closes without need for truncation under the influence of disk self-gravity (Wilson, 1981). Its full characteristics and extensive reference list cannot be covered in this brief account, but an important ingredient to overall logic is that Accretion (companion star to disk and then to disk's central star) and Decretion (central star to disk) occur together or over short cycle times - thus leading to the designation A-D disk. The A-D concept may apply to CVs and W Sers and leans on knowledge about Be stars and $\beta$ Lyr. It is not likely to be useful for optically thin disks, but semi-transparent edges could be interesting. Most light curve analyses for binary circumstellar disks have been based on a non-SG, entirely opaque, axisymmetric model. In contrast, the A-D model naturally produces phenomena that are characteristic of SG disks in binaries, in particular tidal variation with gravity brightening and an outer null-gravity point whose location can now be computed accurately (Wilson, 2018).

Why might structurally significant disk mass be seriously entertained, considering that massive disks are seldom mentioned? One reason is that substantial mass helps a disk to survive nova explosions that would blow a fluffy disk away. The only blast wave particles likely to be present in substantial quantity and not blocked by a disk that is thoroughly optically thick are neutrinos - everything else that impinges on the disk communicates its kinetic and thermal energy to the disk, so the problem essentially simplifies to energetics alone. The issue is just whether the added energy augments the disk's orbital energy to positive values. Fig. 7 of Wilson (2018) illustrates results for seven explosion energies from $10^{43}$ to $10^{47} \mathrm{erg}$ and 17 disk masses from $10^{-5}$ to $10^{-1} M_{\odot}$, assuming that 
the disk intercepts $40 \%$ of the blast geometrically. So do the disks of classical novae survive the explosions? Only novae that are observed both before and after eruption can tell us - realistically this has meant very few. However recurrent nova CI Aql was so observed by R. K. Honeycutt (Wilson \& Honeycutt, 2014) with the light curve and overall brightness being about the same before and after, as illustrated by comparison of figs 5 and 6 of Wilson (2018). Accordingly, the disk - by far the brightest light source in the system and the only cause of eclipses of the mass donor star - was not blown away.

\section{Where might massive disks reside?}

Large amounts of transferred mass favor major spin-up of an accreting star and also favor having significant accumulated mass in a disk. A few percent - perhaps as little as $1 \%$ of the accreting star's mass - could be structurally sufficient, as shown by numerical experiments of Wilson (1981). W Serpentis stars (like $\beta$ Lyr) have large mass-transfer rates and classical novae transfer at low rates for long times. Accordingly, W Sers, classical novae, and novalike variables are candidate locales. How can a massive disk be recognized? Figs 10 and 11 of Wilson (2018) show dependence of disk thickness on disk mass and several other parameters, although quantitative analysis is needed to distinguish among several parameters that affect disk thickness and other disk characteristics. However, serious observational problems stand in the way of useful disk mass determinations. These include wild light curve excursions owing to disk disturbances, novae being mainly observed only in and just after outburst when disks are most disturbed, and the mass-receiving stars in many systems being hidden within the disk, so unobservable.

\section{Impersonal results by Differential Corrections}

The CI Aql and $\beta$ Lyr solutions of Wilson (2018) were by subjective (trial and error) adjustments, while objective solutions based on a weighted Least Squares criterion can now be done with an extension of the Differential Corrections (DC) program that is part of the public W-D model (Wilson \& Devinney, 1971; Wilson, 1979, 1990, 2008; Wilson \& Van Hamme, 2014). Results are in Table 1, where $a=a_{1}+a_{2}$ is the semi-major axis of the relative binary orbit, $F_{1}$ is the ratio of star 1's surface angular rotation to the orbital angular rotation, $\rho_{\text {disk }}$ is mean disk density over optically visible depths, $u_{\text {wire }}$ is the radius of the wire-like mass concentration, $x_{\text {outer }}$ is the $x$ coordinate of the disk's outer surface, $x_{\text {null }}$ is the outer null effective gravity $x$ coordinate where material becomes unbound to the disk (see section 5.9 of Wilson $(2018)$ ), $x_{\max }$ is the x-coordinate of greatest disk thickness, and $z_{\max }$ is the disk half-thickness at $x_{\max }$. See section 6.4.1 of Wilson (2018) for definitions of computed angular velocity exponents $n_{\text {inner }}$ and $n_{\text {outer }}$. Quantities without standard errors were adopted from the literature 
Table 1. Parameter Results from CI Aql DC Solution

\begin{tabular}{lc|lc}
\hline \hline$a$ & $4.5719 R_{\odot}$ & $T_{\text {eff }}^{\text {disk }}$ & $15729 \pm 785 \mathrm{~K}$ \\
$P_{\text {orb }}$ & 0.6183619 & $\rho_{\text {disk }}$ & $3.43 \pm 0.17 \times 10^{-11} \mathrm{~g} \mathrm{~cm}^{-3}$ \\
$F_{1}$ & 6000 & $M_{2} / M_{1}$ & 2.50 \\
$i$ & $67.49 \pm 0.45$ & $M_{\text {disk }} / M_{1}$ & $0.0325 \pm 0.0037$ \\
$T_{\text {eff }, 1}$ & $15000 \mathrm{~K}$ & $u_{\text {wire }} / a$ & $0.044 \pm 0.011$ \\
$T_{\text {eff }, 2}$ & $6100 \mathrm{~K}$ & $x_{\text {outer }} / a$ & $0.212 \pm 0.013$ \\
$M_{1} / M_{\odot}$ & 0.96 & $R_{1} / R_{\odot}$ & 0.0069 \\
$M_{2} / M_{\odot}$ & 2.42 & $R_{2} / R_{\odot}$ & 2.04 \\
$n_{\text {inner }}$ & -1.493544 & $x_{\text {null }} / a$ & 0.416547 \\
$n_{\text {outer }}$ & -1.894034 & $x_{\max } / a$ & 0.17134 \\
$L_{1} /\left(L_{1}+L_{2}\right)_{V}$ & $1.44 \pm 0.12 \times 10^{-4}$ & $z_{\max } / a$ & 0.15411 \\
\hline \hline
\end{tabular}

without adjustment. As DC's numerous and thoroughly tested provisions for performance enhancement are already in place within the disk-revised version, expectations for reliable disk model solutions from the start appear to be realistic. This paper's DC solution is compared with a subjective light curve fit from Wilson (2018) in Fig. 2.
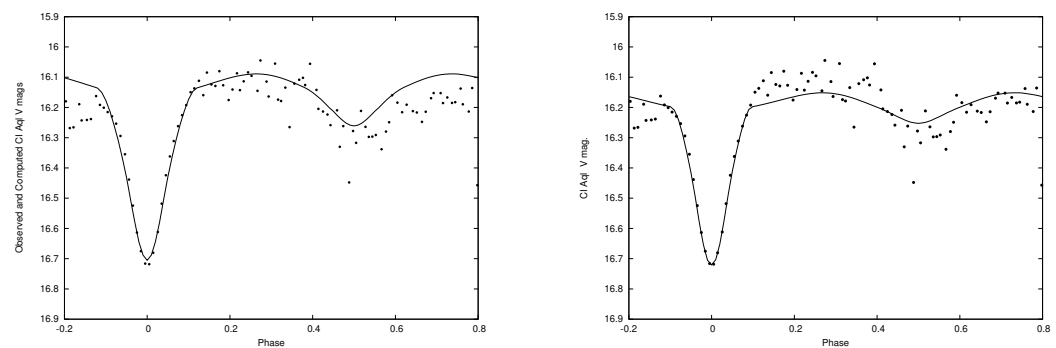

Figure 2. Comparison of trial and error light curve fit (Wilson, 2018) (left panel) and this paper's impersonal (DC) solution (right panel). The impersonal solution splits the height difference between the maxima, rather than favoring maximum I, and represents the eclipse of the disk noticeably better. It also supplies standard error estimates.

Acknowledgements. Discussions with Walter Van Hamme, along with several other kinds of expert help, are greatly appreciated.

\section{References}

Çakirli, Ö., Ibanoglu, C., Sipahi, E., et al., Analysis of the massive eclipsing binary V1441 Aql. 2015, New Astr., 34, 15

Hachisu, I., Kato, M.: 1999, Astrophys. J. 517, L47

Kopal, Z., The classification of close binary systems. 1955, Ann. d'Ap., 18, 379 
Kopal, Z. 1959, Close Binary Systems

Kuiper, G. P., On the Interpretation of $\beta$ Lyrae and Other Close Binaries. 1941, Astrophys. J., 93, 133

Linnell, A.P., Hubeny, I.: 1996, Astrophys. J. 471, 958

Linnell, A. P., Harmanec, P., Koubský, et al., Properties and nature of Be stars. 24. Better data and model for the Be+F binary V360 Lacertae. 2006, Astron. Astrophys., 455, 1037

Lucy, L. B., The Structure of Contact Binaries. 1968a, Astrophys. J., 151, 1123

Lucy, L. B., The Light Curves of W Ursae Majoris Stars. 1968b, Astrophys. J., 153, 877

Lucy, L. B., The Common Convective Envelope Model for W Ursae Majoris Systems and the Analysis of their Light Curves. 1973, Astrophys. Space Sci., 22, 381

Mauder, H., Investigations on the structure of W UMa stars. 1972, Astron. Astrophys., 17,1

Mochnacki, S. W. \& Doughty, N. A., A model for the totally eclipsing W Ursae Majoris system AW UMa. 1972, Mon. Not. R. Astron. Soc., 156, 51

Mochnacki, S. W. \& Doughty, N. A., Models for five W Ursae Majoris systems. 1972, Mon. Not. R. Astron. Soc., 156, 243

Palma, T., Minniti, D., Dékány, et al., New variable stars discovered in the fields of three Galactic open clusters using the VVV survey. 2016, New Astr., 49, 50

Rucinski, S. M., The W UMa-type Systems as Contact Binaries. I. Two Methods of Geometrical Elements Determination. Degree of Contact. 1973, Acta Astron., 23, 79

Rucinski, S. M., Binaries. II. A- and W-type Systems. The W UMa-type Systems as Contact. 1974, Acta Astron., 24, 119

Terrell, D., Understanding Overcontact Binaries. 2005, J. American Assoc. Variable Star Observers 34, 103

Terrell, D. \& Nelson, R. H., The Double Contact Nature of TT Herculis. 2014, Astrophys. J., 783, 35

Wilson, R. E., Eccentric orbit generalization and simultaneous solution of binary star light and velocity curves. 1979, Astrophys. J., 234, 1054

Wilson, R. E., Equilibrium figures for Beta Lyrae type disks. 1981, Astrophys. J., 251, 246

Wilson, R. E., Accuracy and Efficiency in the Binary Star Reflection Effect. 1990, Astrophys. J., 356, 613

Wilson, R. E., Binary Star Light-Curve Models. 1994, Publ. Astron. Soc. Pac., 106, 921

Wilson, R. E., Binary Star Morphology and the Name Overcontact. 2001, Inf. Bull. Variable Stars, 5076, 1 
Wilson, R. E., Eclipsing Binary Solutions in Physical Units and Direct Distance Estimation. 2008, Astrophys. J., 672, 575

Wilson, R. E., Self-gravitating Semi-transparent Circumstellar Disks: An Analytic Model. 2018, Astrophys. J., 869, 19

Wilson, R. E. \& Devinney, E. J., Realization of Accurate Close-Binary Light Curves: Application to MR Cygni. 1971, Astrophys. J., 166, 605

Wilson, R. E. \& Devinney, E. J., Fundamental data for contact binaries: RZ Comae Berenices, RZ Tauri, and AW Ursae Majoris. 1973, Astrophys. J., 182, 539

Wilson, R. E. \& Honeycutt, R. K., Outburst-related Period Changes of Recurrent Nova CI Aquilae. 2014, Astrophys. J., 795, 8

Wilson, R. E. \& Van Hamme, W., Unification of Binary Star Ephemeris Solutions. 2014, Astrophys. J., 780, 151

Wilson, R. E., Van Hamme, W., \& Pettera, L. E., RZ Scuti as a double contact binary. 1985, Astrophys. J., 289, 748 